\title{
Prof. Dr. Gerhart Hitzenberger zum 90. Geburtstag
}

\author{
Herbert Kurz · Johannes G. Meran · Peter Mikosch · Peter Pietschmann
}

(C) Springer-Verlag GmbH Austria, ein Teil von Springer Nature 2019

Herr Professor Gerhart Hitzenberger ist Ehren-Redakteur der Wiener Medizinischen Wochenschrift (WMW). Aktiv leitete er die WMW von 1978 bis zum Jahr 2000.

Als aktuelle Herausgeber der WMW fühlen wir uns den Prinzipien, die Gerhart Hitzenberger zeit seines Wirkens als Wissenschaftler, Arzt und Chefredakteur verfolgt hat, verpflichtet: Neugierde auf Neues, wissenschaftliche und menschliche Redlichkeit und Transparenz in Prozessen und in der Kommunikation. Mit Ausdauer und Begeisterung hat er das älteste noch erscheinende deutschsprachige wissenschaftlich-medizische Journal durch viele Jahre gelenkt und an uns heutige Redakteure übergeben. Wir sind dankbar für seine Arbeit und stolz darauf, die WMW 2020 in ihr 170igstes Jahr führen zu können.

Prof. Hitzenberger, lieber Gerhart, Vorbild und Freund, wir wünschen Dir von Herzen alles Gute zum Geburtstag!
Die Herausgeber

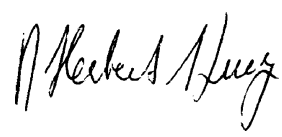

Herbert Kurz

D. Aehours Whens

Johannes G. Meran

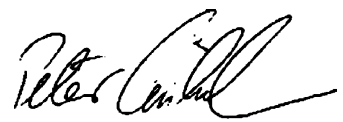

Peter Mikosch

Mn Veken teibilem

Peter Pietschmann

Interessenkonflikt H. Kurz, J.G. Meran, P. Mikosch und P. Pietschmann geben an, dass kein Interessenkonflikt besteht.

Hinweis des Verlags Der Verlag bleibt in Hinblick auf geografische Zuordnungen und Gebietsbezeichnungen in veröffentlichten Karten und Institutsadressen neutral. 